\title{
LV. On peculiarities observed in iron and steel at a bright-red heat
}

\section{H.F. Newall M.A.}

To cite this article: H.F. Newall M.A. (1887) LV. On peculiarities observed in iron and steel at a bright-red heat , Philosophical Magazine Series 5, 24:150, 435-439, DOI: 10.1080/14786448708628124

To link to this article: http://dx.doi.org/10.1080/14786448708628124

曲 Published online: 29 Apr 2009.

Submit your article to this journal $[\pi$

Џ Article views: 2

Q View related articles $₫$ 
LV. On Peculiarities observed in Iron and Steel at a BrightRed Heat. By H. F. Newald, M.A., Demonstrator of Experimental Plysics, Cambridge University*.

T $N$ the September number (p. 256) of the Philosophical 1 Magazine, which I am sorry not to have seen till last week (Oct. 6), there appears a paper by Mr. Herbert Tomlinson on a "Remarkable Effect on raising Iron when under Temporary Stress or Permanent Strain to a Bright-red Heat." I have been engaged for some time past on experiments bearing on the subject of his paper, and as I hope before long to be able to publish a full account of my experiments, I will not at present do more than put down a few notes on such of the more definite results that $I$ have obtained as seem to throw light on the interesting phenomena recorded in Mr. Tomlinson's paper.

First I will describe what I observe as to Reglow or Recalescence. A wire (about $1 \mathrm{~mm}$. diam.) steel or iron, of certain composition, is heated gradually by a strong current or in the flame of a Bunsen burner; it becomes luminous ; the luminosity increases as the temperature rises, until a certain point is reached, when in some cases it pauses in its increase, in others it actually diminishes : this I will refer to as the Darkening; and then it increases again until the wire melts. However, let the beating be stopped before melting takes place and let the wire cool gradually, or, rather, let it be placed in such circumstances as one would naturally expect would promote gradual cooling : the luminosity decreases until a certain point is reached, roughly speaking about dull red heat, when it ceases to decrease and in some cases only pauses, in others it actually increases: this is spoken of as the Reglow; and then it diminishes again until the wire is no longer luminous.

I am still working with a view to finding out to what differences in composition are to be attributed the differences observed in the effects just described. I am fortunate enough to have enlisted the interest of Col. Dyer, of Lord Armstrong's firm, and of Mr. Jenkins, of the Consett Iron Works, and of Mr. Firth, of Sheffield, who have most kindly supplied me with many specimens of steel of known composition. After a great many experiments and observations,-many of them merely careful repetitions and extensions of Gore's results and of Barrett's, many of them, however, new,-I

* Communicated by the Author. 
incline to regard the change going on in iron and steel at high temperatures as partaking of the nature of an explosion, in that once started it continues throughout the mass of the iron, and is evidenced amongst other phenomena by the reglow. Until the "darkening" has taken place the iron is not in a state fit to explode, so to speak ; and hence it is necessary to raise the iron above this temperature in order that reglow may be possible. But before the iron can be raised above this temperature, heat must have passed into it in sufficient quantity to separate the elements producing the explosion. My evidence for this view of the matter I hope to publish in full.

Briefly, I have shown that reglow is not due to chemical action at the surface of the iron; that it is not due to occlusion of gases ; that it is not due to differences in conductivity in iron ai different temperatures, as suggested by Forbes; that there is a rise of temperature not only at the surface, as has been shown by Barrett, but also throughout the mass; that this rise of temperature will partly account for peculiarities(1) in the thermoelectric properties, observed by Tait ; $(2)$ in the electrical conductivity, observed by Smith, Knott, and Macfarlane ; (3) in the thermal expansion, observed by Gore and by Barrett; (4) in the rigidity, observed by Tounlinson and independently by myself ; (5) in the viscosity, observed by myself, and probably by Barus and Stroubal, though I cannot find mention of it ; (6) in the possibility of hardening, observed by myself ; (7) in the return of magnetic properties, recorded by many observers.

My present aim is to find out the cause of this rise in temperature; and my belief is that it is an internal chemical action; and the question is, What is this action? I hope to clear the matter up by experimenting upon specimens of known composition. So far as I have gone I have come across very curious differences in the effects shown by different chance specimens. In some specimens the reglow takes place much more leisurely than in others ; sometimes it is practically a flash, and at other times it is not perceptible at all with the most careful observation. I reserve a detailed account of my observations, and at present will only mention in this connexion that $I$ am not inclined to attribute any important part in the phenomena to the appearance of magnetic properties; and I realize how, if my view of the cause of reglow is correct, a difficult field for inquiry is opened out, necessitating the production of evidence that the properties of iron are different above and below the temperature of the reglow phenomena, or, rather, that the temperature-coefficients 
of the various physical properties suddenly change at that critical temperature.

I will now return to the subject of Mr. Tomlinson's paper; and as I have independently made observations on similar points, I will give my view of the case, as applied to Mr. Tomlinson's experiments. In Experiment I. a wire was heated under torsional stress, and when a certain temperature was reached the rigidity gave way somewhat suddenly and the wire was permanently twisted. "The slight check in the rate at which the wire was permanently twisting" is to be attributed to a similar check in the rate of decrease of rigidity at the "darkening." The wire was allowed to cool ; the rigidity increases until the reglow takes place, and this involves a sudden rise in temperature with corresponding fall in rigidity, and the wire twists further.

I have been nonplussed by what appears to be an inconsistency in the results recorded in Experiments I. and II. In Experiment I. the stress produces permanent strain ; in II. a very feeble stress (magnet deflected through $10^{\circ}$ ) produces a permanent strain; but an intermediate stress (magnet deflected through $90^{\circ}$ ) is not stated to have produced anything but a temporary effect at the higher temperature; and this effect-untwisting during the heating of the wire-is such as to overcome the external stress, as if we had to deal with some instability. I hesitate to attempt an explanation of the first part of Experiment II. ; the second part admits of an attempt, suggesting further tests.

In Experiment II. (second part), then, we have to deal with the case of a wire under very feeble external stress without permanent strain; as the temperature rises there is a sudden untwist, that is, the needle is deflected further from the meridian, and also permanent twist ; and as the wire cools, there is a sudden temporary twist at nearly the same temperature. I think it possible to explain the untwist by attributing it to a pause in the permanent twisting, resulting in a swing of the magnet temporarily from the meridian; the pause being due to the fact that the "darkening" is taking place, and with it a pause in the decrease of rigidity. It would be interesting to repeat this experiment with a view to testing how far all the movements of the needle actually represent stresses in the wire.

Experiments IV. and V. are of great interest as showing both the effect of permanent stress ( $I$ leave out of the case the magnetic effects observed), and the failure of a very common method of annealing.

With permanent torsional strain are introduced internal 
torsional stresses, which differ not only in magnitude but even in direction in different annuli of the wire's section when the external stress is removed; that is to say, il' we regard the wire in this condition as divided into two parts, a core and an enclosing tube or rind, we may choose the radius of the core so that the internal stresses in the core are opposed to those in the rind. If by any means we can weaken the rigidity or elasticity in one part more quickly than that in the other, it is clear that we should get the permanent strain either increased or diminished accordingly. Here, then, we have another way in which the results recorded in $\mathrm{Mr}$. 'Tomlinson's paper, "On the Effect of Change of Temperature in Twisting or Untwisting Wires" (p. 253), may be regarded. I will not dwell upon these, but will deal with Experiment IV., in which a wire permanently twisted is heated, and shows twist in heating and untwist in cooling. We have then to show that in heating the inner core is heated more quickly than the outer rind, in which case the wire will untwist. This case presents little difficulty, except in that at one point during the heating the core may be cooler than the rind, namely, if the wire shows "darkening," with a fall of temperature. Next we have to show that at a point in the cooling the outer rind can become hotter than the core. Now in cooling, the rind reaches the temperature at which reglow occurs before the core; and so it is possible that the rind may become considerably hotter than the core, its rigidity also becoming less, and the wire therefore is untwisted. The suddenness of the twist or untwist depends on the shortness of the period (in both heating and cooling) during which the rigidities of the core and rind differ inarkedly. At low temperatures the rigidity apparently does not vary much with temperature, or at least there is not much greater variation of rigidity in the permanently strained rind than in the core, and the internal couples are almost balanced. But at a certain temperature the rigidity suddenly falls, as is shown by Experiment I., and the balance of couples in the unequally heated wire is destroyed until the wire is heated so much above this critical temperature that an approximate balance of weaker couples is again established. If the temperature of the wire is kept at about a dull red heat (as seen from outside), it is possible to keep the balance of couples so far disturbed as to get nearly all the permanent twist out of the wire.

The explanation above suggested is based on (1) the fact that the reglow is connected with a rise of temperature-this 
is established by my own experiments ; (2) the fact that there is a sudden fall in rigidity at a temperature about red heat--this is established by Mr. Tomlinson's Experiment I. as well as by similar experiments of my own; (3) the assumption (? fact) that a wire of even $1 \mathrm{~mm}$. diam. is not heated uniformly throughout its thickness, but that the inner part is hotter than the parts near the surface ; and lastly that the rigidity of the iron at the high temperature of reglow is less than the rigidity of iron at not so high a temperature before reglow.

A similar line of argument will explain the results recorded in Experiments VI. and VII.

I have made a great number of experiments on the return of magnetic properties in cooling iron; and can at present only say with certainty that the matter is not so simple as appears from Experiment IV. of Mr. Tomlinson's paper. The relation between the reglow and return of magnetic properties varies with the specimens of iron and steel used. I have found some few specimens in which the reglow takes place in the middle of the return of magnetic properties, so that the galvanometer indication is two kicks, the first very small, just before reglow, the second and main kick after reglow, as if the metal was becoming magnetic, when the rise of temperature, coincident with reglow, caused it to pause for an instant. In general, however, the reglow precedes the return of magnetic properties, though by intervals which vary with the specimens observed. I hope to have more definite results when I have tested the numerous specimens of steel and iron which $I$ have now in my possession.

LVI. On "Random Scattering" of Points on a Surfuce. By Joseph KleIBer, Privat-Docent in the Imperial University of St. Petersburg. ${ }^{*}$

TN a paper published in the Philosophical Magazine [ser. 3], 1 vol. xxxvii. pp. 401-427 †, Prof. Forbes set forth some objections against the application of the principles of the mathematical theory of probabilities to the question of the distribution of stars in the sky. Mr. Todhunter quotes this paper in his well-known ' History of the Theory of Probabi-

* Communicated by the Author.

$\dagger$ "On the Alleged Evidence for a Physical Connexion between Stars forming Binary or Multiple Groups, deducte from the Doctrine of Chances." 